\title{
La religión bajo la perspectiva evolucionista de Daniel Dennett*
}

\author{
Alberto Ramírez Téllez
}

Recibido: 13 de marzo de 2015 - Revisado: 21 de abril de 2015 Aprobado: 8 de mayo de 2015

\section{Resumen}

La intención que guía este trabajo es caracterizar la posición que Dennett, desde su perspectiva de la teoría evolutiva, asume frente a la religión. Bajo una metodología descriptiva este artículo da a conocer el escenario desde el cual Dennett estudia la religión en medio de grandes avances científicos y del resquebrajamiento de las formas institucionales, entendiendo que, si bien su crítica, por darse fuera de las líneas rectoras de una reflexión tradicional del fenómeno religioso, puede parecer infundada, ella va a incidir, se quiera o no, gracias a la gran divulgación del pensamiento de Dennett, en la percepción que van a tener no solo las personas de la calle, sino incluso los nuevos teóricos de la religión. La crítica de Dennett a la religión no agota sin embargo, una gran convicción: si bien la persona sale del mundo, de la materialidad y de la animalidad, se levanta y emerge por encima del mundo de la cultura y de la libertad en busca de su último sentido, que merece ser llamado Dios.

Palabras clave: evolución, religión, ciencia, mundo.

* $\quad$ Artículo de investigación que presenta resultados del proyecto doctoral en filosofía en Universidad Nacional de Educación a Distancia (UNED), España. DOI: http://dx.doi.org/10.15332/s0120-8454.2015.0087.06

** Doctorando en Teología, Universidad de Ratisbona (Alemania), en Filosofía en la Hochschule für Philosophie (Múnich, Alemania) y en la Universidad Nacional de Educación a Distancia (UNED), España. Dirige el Departamento de Humanidades y Formación Integral de la Universidad Santo Tomás, Bogotá. Dirección postal: Cra. 9 51-11, Bogotá (Colombia), correo electrónico: frayalbertoramirez@usantotomas.edu.co. 


\section{Religion under the evolutionary perspective of Daniel Dennett*}

Alberto Ramírez Téllez

\section{A bstract}

This work intends to characterise Dennett's position regarding religion from the perspective of evolution theory. With a descriptive methodology, this work demonstrates the perspective from which Dennett studies religion, which today finds itself exsiting alongside great scientific advances and insititutional crises. Although Dennett's critique is somewhat outside of the traditional reflection with regards to religious phenomenon, it will gain influence regardless of whether that be the wish of everybody; it will have an effect not only on everyday people but also new religious theorists. However, the critique that Dennett makes of religion will not destroy a fundamental conviction: although people are beings born of their worlds, and from an animal condition, they will rise and emerge above their culture and freedom in search of the most profound sense of life, which could well be called God.

Keywords: Evolution, religion, science, world.

Research article presents results of the doctoral project in philosophy at the Universidad Nacional de Educación a Distancia (UNED), Madrid, Spain.

** Ph. D. student of Theology, University of Regensburg (Germany), in Philosophy at the Hochschule für Philosophie (Munich, Germany), and in Universidad Nacional de Educación a Distancia (UNED), Spain. Directs the Department of Humanities and formation of the University of St. Thomas, Bogotá. Address: Cra 9 51-11, Bogota (Colombia), email: frayalbertoramirez@usantotomas.edu.co. 


\section{La religion sous la perspective évolutionniste de Daniel Dennett*}

Alberto Ramírez Téllez

\section{Résumé}

L'intention qui guide ce travail est de déterminer la position que Dennett, à partir de son point de vue de la théorie de l'évolution, assume face à la religion. A partir d'une méthodologie descriptive, cet article révèle le scénario à partir duquel Dennett étudie la religion au sein de grandes avancées scientifiques et de la rupture des formes institutionnelles, en comprenant que, si bien sa critique, par le fait de se trouver en dehors des lignes directrices d'une réflexion traditionnelle du phénomène religieux, peut paraître sans fondement, elle aura un effet, voulu ou non, grâce à la grande divulgation de la pensée de Dennett, dans la perception que vont avoir non seulement les gens du commun, mais aussi les nouveaux théoriciens de la religion. La critique de Dennett envers la religion, ne laisse cependant pas de côté une grande conviction: si bien la personne sort du monde, de la matérialité y de l'animalité, celle-ci se lève et émerge au-dessus du monde culturelle et de la liberté à la recherche de son dernier sens, qui mérite d'être appelé Dieu.

Mots clés: Évolution, religion, science, monde.

* $\quad$ Article de recherche présente les résultats du projet de doctorat en philosophie à la Universidad Nacional de Educación a Distancia (UNED), Espagne.

** Doctorat en Théologie, Université de Regensburg (Allemagne), en philosophie à la Hochschule für Philosophie (Munich, Allemagne) et en la Universidad Nacional de Educación a Distancia (UNED), Espagne. Dirige le Département des sciences humaines et de la formation de l'Université de St. Thomas, Bogotá. Adresse: Cra 9 51-11, Bogota (Colombie), e-mail : frayalbertoramirez@usantotomas.edu.co. 


\section{Introducción}

El misterio de la vida, de su origen y de su razón de ser, no es algo que se revela de golpe. Del mismo modo que la formulación de la ley gravitacional nos ha dejado atisbar el misterio del universo, existe una formulación teóricocientífica que nos ha permitido advertir ciertas condiciones en el ascenso de la vida, desde su manifestación biológica más simple hasta su compleja estructura en el ámbito de lo social, se trata de la teoría de la evolución. Esta teoría se comporta hasta hoy a la manera de un principio unificador de la biología. El científico genético y zoólogo Theodosius Dobzhansky llegó incluso a asegurar que "en biología nada tiene sentido, si no es a la luz de la evolución" (citado en Bascompte y Luque, 2012, p. 19) ${ }^{1}$. Hasta hoy, la teoría de evolución no solo es una de las teorías más sólidas, sino incluso más debatidas, que tuvo en las investigaciones -que cumplen ya siglo y medio- de Charles Robert Darwin su expresión más brillante y completa. La teoría darwiana, como sostiene Javier San Martín Sala, "con correcciones, complementos y nuevos descubrimientos, se mantiene como paradigma insustituible en nuestros días, siendo un punto de referencia fundamental par la imagen del ser humano en la actualidad" (San Martín, 2013, p. 236).

La teoría de la evolución, no obstante, requiere espacios de tiempo que exceden considerablemente las posibilidades de una verdadera comprensión por parte de la inteligencia humana. Por esta razón resulta ser, de entrada, una teoría que ha sido muy debatida, más que en el terreno de la comunidad científica, en todos los estamentos de la vida social: para algunos refutada o superada y para otros reivindicada y confirmada (Ayala, 1991), ella siempre dará de qué hablar. Sin embargo, lo que aquí interesa primordialmente no es este debate, sino tener en consideración aquellos postulados fundamentales de la teoría evolutiva que jugarán un papel predominante en el examen del concepto de religión en el pensamiento de Daniel Dennett. En este sentido, la teoría de la evolución conlleva no solo una estrecha relación entre el mundo físico y el mundo biológico, que da cuenta del proceso irreversible de la materia a la vida de los organismos, sino que, a la vez, introduce una importante y esencial relación de estos mundos con el hombre en toda su extensión y complejidad. Y esa es la principal ganancia de esta investigación: recorrer las correlaciones que se trazan entre el fenómeno religioso -que entiende el hecho religioso en su totalidad $-^{2}$ y el proceso evolutivo, sin que implique, en modo alguno, que esta correspondencia explique la razón de ser de la religión (Gómez, 2007, p. 33).

1 Theodosius Dobzhansky publicó un artículo bajo este título, "Nothing in Biology makes sense except in the light of Evolution", en The American biology teacher (1973, pp. 125-129).

2 Siguiendo a Juan Martin Velasco, es conveniente decir que el abordaje de la religión que se hace en este trabajo se da bajo las condiciones de la fenomenología de la religión, que centra su atención no tanto en la materialidad de los cultos (ritos, sistema de creencias, objetos y personas), sino en aquello que subyace y da significado a estas manifestaciones en un ámbito peculiar de la realidad, que no puede ser subsumido en otros ámbitos posibles como el ético, el psicosocial o el biocultural, entre otros (Fraijó, 1994, p. 70). 
La teoría evolucionista ofrece garantías suficientes para explicar y entender procesos como el paso de lo inorgánico a lo orgánico y la evolución de la vida, pero también ofrece posibilidades para tratar de entender las notas características de lo que hace al ser humano único, como por ejemplo: la apertura a la trascendencia, la libertad, la conciencia y la responsabilidad, entre otras tantas. La evolución, como afirma Gómez Caffarena, significa en este caso "progreso" (2007, p. 33).

\section{La religión y la ciencia en el ámbito de la fillosofía de la religión}

La reflexión en torno al fenómeno religioso tal como lo expresa Dennett y su relación con los otros campos del saber se apoya fundamentalmente en las posibilidades que ofrece la filosofía de la religión, que, como señalan R. Schaeffler (1983, p. 105) y Fraijó (1994, p. 38), no es una teología camuflada ni una especie de creación espuria entre filosofía y teología, sino una disciplina que habla la misma verdad que la teología, pero apelando únicamente a las posibilidades que la razón ofrece. Como bien apunta Juan Antonio Estrada:

La revolución científico-técnica no solo ha transformado económica e institucionalmente a la sociedad, generando una productividad sin precedentes, sino que ha cambiado nuestras categorías culturales y nuestras formas de ser. La razón científica se ha convertido en Occidente en el modelo fundamental del saber, en la razón sin más. Es un nuevo régimen o forma de conocimiento, que determina incluso lo que podamos pensar y cómo podemos enfocar la realidad. Para afirmar coloquialmente que algo es verdadero, decimos que es científico. Y esto equivale a afirmar que todo conocimiento tiene que tener una base empírica para ser cierto. Solo podemos afirmar aquello que es comprobable y experimentable, aquello que puede ser testificado por la realidad, probado o, por lo menos, capaz de ser cuestionado. Es decir, solo podríamos afirmar un ser divino, si hubiera algo que lo hiciera verificable o inviable, si algún hecho pudiera confirmarlo o desmentirlo (Estrada, 2015, p. 47).

El choque de los grandes cambios científicos con la religión fue mucho más dramático por dos motivos: primero, el poder institucional y del orden establecido, otorgado por la tradición milenaria de la que era portadora, dada la experiencia y sabiduría acumulada, y del cual, mal o bien, su autoridad era garante; y segundo, por la cercanía, o mejor, por la armonía entre las verdades teológicas y las leyes por las cuales el mundo todo era regido. Cambiar los parámetros de la ciencia implicaba, necesariamente, afectar la armonía que había sido establecida sobre la base del precepto teológico. Esto lo entendía el cardenal Bellarmino cuando, sobre el caso Galileo, escribía al teólogo carmelita Antonio Foscarini diciendo que si se afirmaban como ciertas las conjeturas de Galileo se pondría en peligro la concepción de las sagradas escrituras. Pero, en definitiva, no eran las 
escrituras y su credibilidad las que se ponían en riesgo. Los avances científicos transformaron por completo el escenario y el horizonte en el que el hombre se movía con confianza. Los senderos que abrían los progresos científicos dieron al mundo y al hombre un nuevo lugar en el cosmos y una clara intuición que respondía al cómo de la existencia.

Si bien el cómo ya no resulta ser para la comunidad científica un enigma, sobre el por qué se sigue guardado aún prudente silencio (Hamburger, 2003; Ortiz, 2008). Y es precisamente esta inquietante pregunta, que interpela tanto a la ciencia como a la religión, un asunto que no estaría bien ni esquivar ni acaparar, cuya aproximación implica un trabajo conjunto, como afirmó Albert Einstein en sus conferencias de 1940 sobre ciencia y religión: "Wissenschaft ohne Religion ist lahm, Religion ohne Wissenschaft ist blind" (citado en Mühlin, 2011, 335), o como diría W. Weisschedel, "si la filosofía deja de interesarse por la religión entra en crisis" (Fraijó, 1994, p. 14). Enfrentar este reto ha generado una discusión que crece en interés y profundidad. $\mathrm{Al}$ respecto no puede pasar desapercibida, especialmente en la segunda mitad del siglo pasado, donde el debate ganó en riqueza e interdisciplinariedad gracias a pensadores, principalmente de origen inglés y norteamericano, como Charles Raven, con su maravilloso trabajo sobre ciencia y religión: Natural religion and Christian theology (1953); Charles Coulson, cultivador de la química cuántica y autor de Christianity in an age of science (1953) y su influyente obra Science and Christian belief (1955); y Ian Barbour, quien escribió Issues in science and religión (1966).

Relevante es igualmente el papel que desde entonces vienen jugando en la relación religión-ciencia una serie de organizaciones y publicaciones a nivel mundial, que mantienen en un alto nivel la discusión. Cabe resaltar, por ejemplo, el Institute for Religion in an Age of Science (IRAS) y el Center for Advanced Study in Religion and Sciences (CASIRAS). Desde 1981 existe el Center for Theology and the Natural Sciences (CNTS), que organiza gran número de cursos y seminarios en distintas partes del mundo y que desde 2003 viene publicando la revista Theology and Science. La revista más antigua sobre este tema es Zygon, publicación compartida por varias organizaciones, entre las que se halla el IRAS. La John Templeton Foundation es quizá una de las fundaciones que más incentivos da, con la implementación de becas y financiación para apoyar investigaciones que desde la ciencias naturales, sociales y humanísticas, aborden cuestiones esenciales que puedan ayudar al progreso de la humanidad. En Europa existe la European Society for the Study of Science and Theology (ESSSAT), que suele realizar congresos y encuentros bianuales desde 1987. Todas estas organizaciones y publicaciones, a la par con un sinnúmero de artículos, congresos, libros y nuevos escenarios de debate, han acrecentado la presión para que junto a los crecientes descubrimientos cosmológicos, físicos, químicos, evolutivos, genéticos y médicos, no se renuncie a la pregunta por el sentido del mundo en el que vivimos, procurando mantener un aporte válido y sólido ante la pregunta por la naturaleza humana en sinergia con los avances genéticos, pero no limitado a ellos. Este trabajo conjunto deja ver, por una parte, cómo la ciencia se abre para entender las percepciones sobre la vida humana que 
brinda la religión y, por otra, cómo el pensamiento religioso en su conjunto, y de manera sobresaliente el cristianismo, permanecen atentos a los desafíos que se abren en el campo científico. Ambas perspectivas se aúnan en su búsqueda del sentido del mundo, de la vida y de lo humano, y brindan la posibilidad de que tanto la religión como la ciencia puedan ser, la una para la otra, discursos inteligibles (Peacocke, 2008, pp. 13 ss.).

Así, pues, la pregunta por Dios y su relación con el hombre es una cuestión relevante para el mundo actual, pero fundamental en la vida de la religión; ella es condición desde la cual el creyente lee la realidad del mundo en el que vive y asume determinados compromisos y comportamientos que necesariamente repercuten en su relación con sus semejantes, con el entorno y con el mundo mismo. La oposición radical a la religión es querer anular la relación del hombre con Dios, considerando que la forma más sencilla es anular la existencia de Dios. Este rechazo a la idea de Dios, que antes de la modernidad parecía un caso aislado, hoy representa una tendencia cada vez más abierta y combativa que se viene manifestando de muchísimas formas, como agnosticismo, apatía, anti-teísmo, librepensamiento, humanismo, irreligiosidad, materialismo, naturalismo, escepticismo o secularismo (Beaman y Tomlins, 2014, p. 1). Muchas de estas tendencias son alentadas con frecuencia desde el seno de la ciencias, en las que el ateísmo pasó de ser mero planteamiento teórico o agnosticismo a convertirse en una crítica abierta justificada en la imposibilidad de que exista un Dios que permita el mal en el mundo y en que la vida de los creyentes o de aquellos que tienen formación religiosa no parece diferir mucho, ni en sus buenos actos ni en sus virtudes, de la de aquellos que no practican ninguna religión. Sin embargo, anular la existencia de Dios no ofrece el resultado esperado por aquellos que consideran la fe en Dios como uno de los principales obstáculos al progreso de la civilización en todo su sentido. La secularización de la edad moderna, que ha podido separar la ciencia de la teología, la iglesia del estado, los valores éticos de los preceptos religiosos, no puede aniquilar, quizá evadir, prohibir o esconder la relación del hombre con Dios (Torres, 2008, pp. 19 ss.). Hoy, ciencia y religión han llegado a un punto en el que es necesario no irse lanza en ristre contra el "oponente", sino abrirse a un diálogo en el que sea posible comprender las razones del otro. Es verdad que estos diálogos no siempre son tan efectivos o no arrojan tan prontamente los resultados deseados, pero siempre serán un canal que ha de mantenerse abierto en el intento por evitar enturbiar la relación (Rahner, 1967).

\section{La propuesta de Daniel Dennett dentro del ámbito transdisciplinario}

Una reflexión seria y conjunta entre religión y ciencia exige actitudes integradoras que permitan una percepción del mundo más amplia y no fragmentada, e implica el deseo conjunto por hallar puntos de contacto, más que las diferencias en el momento de acometer una empresa. La interdisciplinariedad reclama en 
primer lugar poner en común aquellos aspectos que parecen problemáticos o limitadores, el árbol categorial con que se aborda su campo de referencia y, algo muy importante, los métodos que se siguen para el desarrollo de la investigación. Ahora bien, no se trata de aunar y homogenizar un saber enciclopédico; cuando se habla de interdisciplinariedad en el caso concreto de la religión, la filosofía y las ciencias naturales y afines, se está enlazando una propuesta transdisciplinaria, es decir, un paso más allá del simple comprender y tolerar "los otros saberes". No es que se procure alcanzar un método común a todas, sino una herramienta eficiente para dilucidar los retos y desafíos que interpelan al hombre de hoy. El trabajo de Daniel Dennett es un ejemplo maravilloso de recurso a la interdisciplinariedad al momento de abordar problemas complejos; su labor como filósofo y científico se ha visto enriquecida por el estudio de la neurociencia, la genética, la inteligencia artificial, la antropología, la psicología y la sociología.

La continua ausencia de teólogos en la interdisciplinariedad, principalmente cuando se aborda la religión y su papel en la evolución y la cultura, es también un ejemplo diciente de lo precarios y desilusionadores que pueden llegar a ser los resultados cuando, o bien no hay un interés muy vivo por este trabajo entre varias disciplinas, o bien es insuficiente el número de pensadores e intelectuales que estén a la altura de las nuevas exigencias que ello representa (Ferrara, 2012). No obstante, destaca el aporte de teólogos como Juan Antonio Estrada, que resaltan el significado antropológico que subyace a la idea de religión. Existe una toma de conciencia, afirma Estrada (2012), "acerca de la importancia de la religión para la evolución de la especie. La creación es una construcción cultural relacionada con experiencias antropológicas fundamentales" (p. 38).

La relación y referencia que esta investigación hace a los ámbitos que se han mencionado como tocantes a la problemática de la religión en el contexto científico se delimitan de modo particular en el señalamiento de la perspectiva que surge en torno a la religión, considerada como un fenómeno natural en el ámbito de la teoría evolutiva, tal como ha sido considerada a partir de Darwin por los científicos y filósofos de la ciencia contemporáneos en lo que compete al desarrollo y significado de la conciencia, la libertad, la moral, la inteligencia social y la religión.

Dennett encarna con su pensamiento filosófico ingeniosamente abarcador la propensión contemporánea de la divulgación científica de las tendencias de las grandes corrientes del pensamiento científico. Sus libros, conferencias, entrevistas, artículos y videos son accesibles fácilmente al "gran público", brindando ideas, presupuestos y prejuicios, que por resultar atrayentes, claros de entender y muy manifiestos, llegan a influir de tal manera que pueden convertirse fácilmente en criterios o presupuestos con los que seguramente, incluso en un ámbito académico, esos temas vayan a ser tratados. Dennett pertenece al grupo de intelectuales que ha optado por la divulgación científica cuya más robusta finalidad es permitir que los conocimientos o los descubrimientos sean conocidos por el mayor número de receptores. La divulgación científica 
o la literatura popular científica se dirige a un público heterogéneo intentando instalar saberes en la vida cotidiana, ganando relevancia en nuestros días. Esta práctica no puede pasarse por alto y menos cuando se entiende que el debate sobre la religión parece darse como una constante. La religión siempre está en el punto de mira de los avances espaciales, biológicos, antropológicos, psicológicos, neuronales, políticos, económicos o de orden público y, por incidencia, en el orden social actual.

Dedicar este estudio al tema de la religión en Dennett muestra que, en su caso, el tema de la religión no es una excepción, pero también que la claridad y certeza con que Dennett ve el proceso evolutivo de la religión, su comportamiento en medio del progreso social y su conveniente desaparición en un futuro no muy lejano, no son del todo acertadas, manifiestas, ni incuestionables. Si bien Dennett sería de la opinión de Nietzsche respecto a que es caro y terrible el precio que se paga cuando las religiones no están en manos del filósofo ${ }^{3}$, no obstante, como indica Fraijó, el tema de la religión es mucho más complejo y exige siempre una complementariedad que nace de los diversos senderos por los cuales la filosofía y la religión pretenden alcanzar la misma cumbre (1994, p. 38). Para llegar a este cometido, una de las condiciones principales que se da en este tercer horizonte de comprensión es el impulso por dejar atrás la tentativa de edificar cuerpos doctrinales sistematizados. La imagen de grandes ideas, desglosadas en árboles categoriales y engranados bajo una articulación impecablemente coherente de una postura intelectual ha quedado atrás. La imagen compleja del mundo que nos rodea obliga a otro tipo de reflexión más amplia, ecuménica y, en muchos casos, como en el particular de Dennett, descriptiva, sin que por ello se tenga que juzgar necesariamente de "poco rigurosa". El mundo que nos rodea difiere mucho del mundo de Platón, Agustín, Tomás, Hegel e incluso del mundo de Husserl $^{4}$ y Heidegger. Al respecto, el profesor de la Universidad del Rosario Carlos Maldonado señala:

Vivimos en un mundo irregular, moteado, imperfecto. La regla no es la regularidad, sino, en términos cotidianos, el anonimato [...] Desde el punto de vista de la ciencia, el mundo ha sido objeto de estudio en términos de fenómenos simples y controlados. Es más, perfectamente controlados en el laboratorio. En numerosas ocasiones -muchísimas, de verdad-, lo que sucede con éxito en el laboratorio (desde la física en general hasta la biología molecular, desde la química hasta la ciencia de materiales, por ejemplo) difícilmente ocurre de la misma manera en el mundo real. No obstante, el sentido de la ciencia y de la cultura consiste en encontrarle sentido a lo que no lo tiene -una preocupación que, en realidad, heredamos del siglo XIX: la preocupación por el sentido-. Así, tanto la ciencia como la cultura -particularmente el arte, en

3 "Es bezahlt sich immer theuer und fürchterlich, wenn Religionen nicht als Züchtungs- und Erziehungsmittel in der Hand des Philosophen, sondern von sich aus und souverän walten" (Nietzsche, 1966, N. 62).

4 "Tatsachen Wissenschaften machen Tatsachenmenschen", dice Husserl en la Crisis de las ciencias europeas y la filosofía fenomenológica (citado en Maldonado, 2012, pp. 7-102). 
su sentido más amplio- buscan, descubren, encuentran patrones, regularidades y leyes en un mundo esencialmente sorpresivo, que sucede, en rigor, a la manera del puntillismo o del impresionismo (Maldonado, 2012, pp. 21 ss.).

En este contexto, ciencia y religión establecen nuevas condiciones en su forma de relacionarse, si bien -como se indicó anteriormente en el caso de Dennett-se parte del presupuesto de desconocer cualquier dato sobrenatural como materia de estudio de la ciencia y de reducir el fenómeno religioso al fenómeno del comportamiento ético del hombre; aparece, no obstante, un aspecto significativo que ayuda a comprender su crítica a la religión, su postura como divulgador y su tendencia a "polemizar" sobre aspectos que siempre generarán debate y una gran audiencia, aún a expensas de que la disquisición resulte o no pertinente. Bien lo decía Fagundes dos Santos:

Al hacer declaraciones polémicas como "Dios no existe", "la religión es perjudicial para la humanidad", "no hay vida después de la muerte ni en otros planetas" entre otras, los fundamentalistas se hacen famosos y ganan espacio en los medios de comunicación. Todos los activistas anti-religiosos se unen a ellos y la ciencia se presenta como la verdad que vino a barrer todas las creencias. Por el bando contrario, los fundamentalistas religiosos se unen para desafiar a los fundamentalistas de la ciencia y presentar la ciencia actual como algo diabólico. Interminables debates surgen en los que nadie quiere oír, solo hablar. Explota así el odio irracional que es incompatible tanto con el amor predicado por la religión como con la razón defendida por la ciencia. Cada fundamentalista agrada a su respectiva audiencia. Todos ganan, excepto la ciencia (Fagundes, s.f., párr. 15) ${ }^{5}$.

Dennett parece encuadrar perfectamente en esta crítica de Fagundes. En su obra se puede apreciar como su actitud parece coincidir, por un lado, con esa tendencia a la respuesta contundente a cuestiones que en la mayoría de las ocasiones no pueden ser demostradas experimentalmente $\mathrm{y}$, por otro, con su tendencia a considerar el conocimiento científico como el criterio último para medir la veracidad de los argumentos, desconociendo todo el proceso histórico en el que se han venido consolidando los saberes y desconociendo los fundamentos que Popper, Kuhn y Feyerabend, entre muchos otros, mostraban que subyacen a los saberes científicos.

5 "Ao fazerem afirmações polêmicas como 'Deus não existe", 'a religião é prejudicial à humanidade', 'não há vida depois da morte e nem em outros planetas' entre outras, os fundamentalistas ficam famosos e ganham espaço na mídia. Todos os ativistas anti-religiosos se juntam a eles e a ciência é apresentada como a verdade que veio varrer todas as crenças. Em contrapartida, os fundamentalistas religiosos se aglutinam para contestar os fundamentalistas científicos e apresentam a ciência como algo diabólico. E surgem debates infindáveis onde ninguém quer ouvir, apenas falar. Explode o ódio irracional incompatível tanto com o amor pregado pela religião como pela razão defendida pela ciência. Cada fundamentalista agrada seu respectivo público. Todos ganham exceto a ciência" (original en portugués, traducción del autor) 
El nuevo paradigma de las ciencias físicas parece alejarse curiosamente de esta tendencia fundamentalista de mostrar la ciencia como cuerpo doctrinal perfecto y acabado, sin que ello signifique un desequilibrio de su rigurosidad. La cuestión de asumir dentro de sus variables aspectos como la inexactitud, lo poco predecible de algunos fenómenos y su rareza hace en el ámbito científico contemporáneo que lo que no sabemos sea más importante que lo que sabemos (Taleb, 2008, p. 25). El rápido avance de la ciencia no se debe, entonces, de manera indiscutible a la demostración de su poder explicativo y de predicción, basados en leyes que parecen confirmar los fenómenos observados (Victoria, 2011, p. 14). Por más que se intente reducir la religión a una descripción concatenada de acontecimientos en la escala evolutiva, es innegable que ella aparece como un suceso que es precisamente un fenómeno, sencillamente porque nadie esperaba que fuera a aparecer.

Hacer una correlación entre el fenómeno religioso con su línea de avance y el fenómeno natural con la línea de evolución biológica es una apuesta problemática. Cualquier lector podrá entender de entrada que el tratamiento que Dennett estaría haciendo de la religión es identificarla con un acontecimiento regular (aunque a veces inesperado), producto de los procesos periódicos (geológicos, climáticos, cósmicos) que acontecen en la naturaleza. La religión como fenómeno natural y producto de la evolución como un proceso cognitivo adaptativo (Beaman y Tomlins, 2014, p. 57) en Dennett, implicará para este estudio exponer aspectos propios de la teoría evolutiva de Darwin, de la teoría de la mente y la conciencia, de la cultura, del valor de la libertad y de lo ético, dentro de los cánones del proceso evolutivo que inicia en lo biológico y se transforma en un fenómeno cultural.

\section{La cuestión de la religión en Daniel Dennett}

Si se preguntara qué entiende Dennett por religión, en un intento por conceptualizarla, se podría decir que la considera ${ }^{6}$ como un fenómeno natural -subordinado al proceso evolutivo biológico (Dennett, 2006/2007, pp. 96-290 ss.; 2009, pp. 531-532) y cultural (2006/2007, pp. 176-188, 407 ss.; 2009, pp. 305-324)- que por medio de la sucesión y transformación genética y memética (2006/2007, pp. 105ss., 118-120) expresa, explica y transforma valores personales, éticos y sociales (2006/2007, pp. 52, 80 ss., 315-354), bajo las condiciones de una libertad (2006/2007, pp. 36, 298, 353, 382; 2009, pp. 195-220) no restringida por una supuesta conciencia (Dennett, 2005/2006, pp. 75 ss.), y cuya veracidad se formula bajo la consigna del cui bono (Dennett, 2006/2007, pp. 88-122, 177-215) 7 .

6 Se mantiene en cursiva lo que se sugiere como una conceptualización que permitirá enmarcar las afirmaciones de Dennett sobre la religión y darles a éstas un cierto orden sistemático, conveniente para la exposición de esta investigación.

7 Según David Thomson, Dennett insiste en que hay que preguntar "Cui bono?" (¿A beneficio de quién?): "The mistake, Dennett believes, comes from thinking of Darwinism as a biological theory rather than as an abstract, algorithmic process. We must always ask, "Cui bono?" -to whose benefit? The idea of the Selfish Gene, that adaptation is for the benefit of the gene, may be appropriate to prehuman evolution, but once the cultural transmission of memes begins, there are new entities that may benefit. Memes create new answer-candidates for the cui bono? Question. Yet does 
A primera vista, parecería que Dennett desempolva las ya ampliamente discutidas teorías entorno a la comprensión de la religión. La idea de la religión como fenómeno evolutivo era una idea desarrollada y defendida por el inglés Herbert Spencer, quien en gran medida consideró que la propuesta evolutiva desencadenaba un mecanismo que se daba de hecho en los organismos vivientes y que como tal debería replicarse obligadamente en los humanos ${ }^{8}$. La propuesta de encarar el darwinismo con las ideales intelectuales de avanzada y de pretender señalarlo como la solución a problemas antiguos era ya, pues, un proyecto que se había intentado realizar siglos atrás (González, 2009, p. 33; Gómez, 2007, p. 27). La condición de la religión como hecho social fue ampliamente trabajada por filósofos como Durkheim" ${ }^{9}$ quien definió la religión como "un sistema solidario de creencias y de prácticas relativas a las cosas sagradas, es decir, separadas, interdictas, creencias y prácticas que unen en una misma comunidad moral, llamada Iglesia, a todo los que la aceptan" (Durkheim, 1982, p. 65).

La condición de la religión como fenómeno natural, como la entiende Dennett, no puede concebirse sencillamente como una contraposición, donde un fenómeno natural podría entenderse, primero como aquello que se da de manera espontánea en contraposición a aquello que es adquirido o preparado para ser recibido artificialmente; $y$, segundo, como algo que ya viene dado en los genes. Si bien la condición natural de la religión se enfoca en el proceso cultural evolutivo que se da a través del lenguaje y de los memes, y no tanto del impacto de lo evolutivo biológico (Dennett, 2006/2007, p. 45; 1990, pp. 177-194; 2013, pp. 274 ss.), no pretende por ello subvalorar el papel que lo evolutivo-biológico tiene en la comprensión de la religión como fenómeno natural. Sólo que quiere acentuar primordialmente el ámbito en el que es mucho más pertinente abordar el tema de la religión dentro de la dinámica evolutiva: el proceso cultural.

Cuando Dennett se refiere a la religión como fenómeno natural, está indicando de manera precisa que no es posible, sin más, "saltar" al concepto y ámbito de lo "sobrenatural" y de lo "trascendental" si estos conceptos no son suscitados a partir de experiencias comprobables, que más que responder a un proceso introspectivo reflexivo o irreflexivo, responden a los procesos evolutivos demarcados por los métodos de la ciencia. Al describir la religión como "fenómeno", Dennett se orienta hacia la forma como la religión se "manifiesta", y no tanto hacia la naturaleza de la religión, lo que dispensa a Dennett de considerar lo que subyace velado tras el fenómeno.

this response not just substitute memetic determinism for genetic determinism? How can there be morality if selves are pushed around by forces beyond themselves, by "principalities and powers"? This objection slips back into some substantialist misconception of the self as a pre-existing entity that memes, secondarily, act upon. For Dennett, selves are memetic entities through and through; they are creations of memes. A self is not a pure, pre-existing subject -a cogito- that secondarily comes to possess cultural goods; it is a cultural being to the core. "... [We are] not just guardians and transmitters of culture, but cultural entities ourselves -all the way" (Thompson, 2009, p. 128).

9 Principalmente en la obra de Émile Durkheim publicada en 1912, Las formas elementales de la vida religiosa. El sistema totémico en Australia. Seguimos aquí la traducción de Ramón Ramos (Madrid: Akal, 1982). En la actualidad existe una nueva traducción de Jesús Héctor Ruiz Rivas, publicada en México en 2012 por el Fondo de Cultura Económica. 
Guiado por las manifestaciones del fenómeno religioso, Dennett halla sorprendente la capacidad que tiene la religión de despertar en las personas un compromiso que puede estar incluso por encima del propio bienestar personal $\mathrm{o}$, incluso, sobre el imperativo biológico de engendrar descendencia: probablemente ha muerto más gente, afirma Dennett, en el valiente intento por proteger textos o lugares sagrados que en el intento por resguardar sus provisiones de alimento o a sus hijos o a sus hogares (2006/2007, p. 23).

En un sentido contrapuesto para el estudio científico, al entender de Dennett, por la imposibilidad de confirmar la esencia de la religión más allá del terreno de los fenómenos naturales y sociales, la religión aparece como un "sistema social cuyos participantes manifiestan creencias en agentes sobrenaturales o en agentes cuya aprobación ha de buscarse" (2006/2007, p, 27). Consideración que ha llevado a que muchos científicos sociales aleguen el carácter irracional de la religión ${ }^{10}$. Dennett prefiere, sin embargo, centrarse en un tema más pragmático, aunque no menos controversial: la relación entre la religión y los postulados morales. Según Dennett,

Muchos creen que el papel más importante de la religión es ser soporte de la moralidad al darle a la gente una razón imbatible para obrar bien: la promesa de una recompensa infinita en el cielo, y (según los gustos) la amenaza de un castigo infinito en el infierno si no lo hacen. Este raciocinio sostiene que sin la lógica divina de la zanahoria y la vara, la gente holgazanearía sin meta alguna o complacería sus deseos más bajos, rompería sus promesas, engañaría a sus esposos, descuidaría sus deberes, etc. (Dennett, 2006/2007, p. 326).

Aunque Dennett topa con una de las relaciones esenciales que a lo largo de la historia aparece en el mundo de la experiencia religiosa y que -como afirma Gómez Caffarena (1996) - puede llegar a ajustarse "hasta el punto de que resulta difícil establecer una frontera entre ambas" (p.40), deja de lado el motivo central por el cual la relación se da y aparece tan estrecha: la experiencia de trascendimiento absoluto -sigo también aquí a Gómez Caffarena-. Esta experiencia se refiere no tanto a la naturaleza de la divinidad que irrumpe en el mundo, sino más bien a su actuar o acontecer en el mundo. Sin este presupuesto, la relación puede verse distorsionada, como de hecho lo presenta Dennett, de tal forma que las prácticas tenidas como exigencias morales no tendrían otra justificación distinta que la de ser tan solo medidas normalizadoras del comportamiento humano. Dennett desplegará, sin embargo, sobre este supuesto la idea del cui bono de la religión; concepto clave en sus planteamientos y al cual suele recurrir frecuentemente en los distintos escenarios en los que surge la pregunta por la razón de ser de la religión. 
Abordar éste y los anteriores aspectos del pensamiento de Daniel Dennett supone para esta investigación un examen y un análisis que partan de un abordaje reflexivo cristiano que -como advierte Hans Küng- no ha de responder a una confesión en particular, sino que ha de estar abierto a una consideración cristiana abierta (1996, p. 11). Para ello son de gran valor los aportes, entre otros teóricos, de dos grandes filósofos españoles de la religión: Manuel Fraijó y José Gómez Caffarena. Sus análisis lúcidos, rigurosos y detallados son un excelente y oportuno recurso para aproximarse sin enardecimiento o animosidad a los juicios de pensadores que como Daniel Dennett abordan el tema de la religión con cierto reparo y en ocasiones con abierta hostilidad ${ }^{11}$. Igualmente significativos son los aportes de Juan Antonio Estrada, otro gran filósofo español de la religión, quien ha estudiado el papel de ésta en el contexto cultural, y el complejo tema de la teodicea.

\section{Conclusiones}

Si bien Dennett es un convencido de que el discurso científico aminora o "debe aminorar" los efectos de la religión en una evolución cultural -no hay que pasar por alto que Dennett es uno de los pensadores contemporáneos convencidos de la degradación del discurso religioso, ya no de manera implícita, sino explícita al estilo de Dawkins, Susan, Goluld y Pinker-, se basa, por lo general, en una inadecuada comprensión de la naturaleza de la religión: en parte por la prevención que sobre los postulados religiosos se tiene en el ámbito científico, en parte también por la misma actitud de aprensión de algunos teóricos de la religión frente a la ciencia, lo que a la postre solo propicia una gran confusión en el ámbito cultural y da lugar, paradójicamente, tanto en el terreno de la ciencia como en el de la fe, al afloramiento de actitudes dogmáticas, esotéricas y autojustificadoras que no pueden asimilar con sentido los nunca antes alcanzados avances en la historia de la humanidad.

Lejos de un arrogante "cientificismo" que aboga por la legitimidad única que tiene el saber o el conocimiento científico, y también lejos de la corrosión propia de los relativismos modernos en que pueden caer muchas de las conclusiones de investigaciones científicas, Dennett mantiene la convicción de que su empresa intelectual se confronta en la realidad, "realidad" amplia que ha de compartir, lo sabe muy bien, incluso con aquellos que profesan una fe y que mantienen sus convicciones apelando a la "realidad" de los fenómenos sobrenaturales.

11 No son pocos los científicos que mantienen una actitud de rechazo frente a la religión y a la idea de Dios. Hawking, ampliamente admirado como uno de los mayores científicos de la historia y autor de Historia del tiempo declara su escepticismo frente a la posible existencia de Dios. Un caso más extremo lo tenemos, por ejemplo, en Dawkins y Weinberg, que son abiertamente hostiles a la religión y como se señala en la obra de Giberson y Artigas "no se disculpan por desear que desaparezca. El relojero ciego de Dawkins argumenta que la evolución explica las maravillas del mundo vivo mucho mejor que la religión. Darwin, dice Dawkins, mostró que Dios no es una hipótesis necesaria para explicar el mundo natural. En consecuencia esto implica que la evolución y la religión son incompatibles, incluso más que el hecho de demostrar que Dios no es imprescindible a la hora de dilucidar los misterios más profundos puesto que esto mismo ya es incompatible con la religión" (Giberson y Artigas, 2007, 316). Steven Weinberg también sostiene una actitud ante Dios negativa, incluso dura, y es bien conocido como un adversario público de la religión. En este terreno es igualmente directo e intransigente (Weinberg, 1996, 226). 
Dennett no busca una armonía entre religión y ciencia que las haga concordar, busca más bien explicar por qué el hombre ha llegado a la religión, por qué ella subsiste a pesar de tantos cambios y parece que permanecerá por mucho tiempo más. Estas preguntas van imponiendo a Dennett, de alguna manera, que, a la par de su comprensión del proceso evolutivo -que explica de manera tan satisfactoria la mayoría de los procesos biológicos-, se pregunte por el origen y la dinámica de conductas humanas que por su complejidad parecen ser conductas irrepetibles en otras especies y parecen no poseer rastros de presencia en estadios inferiores de la evolución. Dichas cuestiones no hacen desistir a Dennett de proclamar su tesis fundamental: la religión es un fenómeno natural que debe ser estudiado como tal y cuya huella ha de rastrearse, como se han hecho en el caso del estudio de otros fenómenos naturales, a través del proceso evolutivo.

Esta tesis, sin embargo, está legitimada en otra no menos importante para Dennett y para un número creciente de científicos de otras áreas del saber: cuando se habla de evolución no se ha de entender tan solo la dinámica de un proceso biológico y de una herencia genética transmitida y nada más, la cultura y la herencia que a través de ella se transmite y se recibe son también elementos intrínsecos y constituyentes de lo que propiamente se ha de entender por evolución. Con este criterio, los aspectos del hecho religioso que Dennett estudia son bastante delimitados y excluyen de entrada la pregunta por la posibilidad de lo sobrenatural en cuanto tal. Los cuestionamientos que Dennett eleva a la religión y el juicio de credibilidad que le aplica, responden de manera casi exclusiva a aspectos que, o bien tienen que ver con el proceso evolutivo, o bien responden a asuntos propios de la dinámica social.

Para un creyente el criterio de Dennett deja por fuera lo "más esencial de la religión" y las preguntas medulares sin contestar; no cabe duda, sin embargo, que su investigación, sus conclusiones y el impacto que todo esto genera son un aporte valioso para que la religión, particularmente la reflexión teológica, perciba la forma como es vista, estudie los resultados expuestos y enfrente con estos conocimientos y presupuestos los desafíos que la cultura científica en particular y la cultura humana en general le presentan al momento de formular y justificar las condiciones en que el hombre se relaciona con Dios, con el mundo y con sus semejantes. Y es que en definitiva, como señala Fraijó con relación al pensamiento de Pannenberg, el hombre está esencialmente orientado hacia la infinitud y lo que más interesa en ese contexto no es ver si la religión ha actuado como fenómeno positivo o negativo, sino su permanencia en el tiempo presente (Fraijó, 1986, p. 147).

La investigación de Dennett se enmarca en un contexto de crisis de la credibilidad institucional en el mundo contemporáneo, que involucra también las instituciones y credos religiosos. Es innegable que el cristianismo en Europa y América ha sufrido un cierto colapso en este sentido, cuando se produce, ante la crítica social, la percepción de que muchas de las instituciones, en otro tiempo depositarias de confianza y credibilidad, no lograron (ni lo harán) satisfacer el lugar que ocuparon en medio de la sociedad en cuanto a coherencia interna, 
fecundidad y, algo muy importante para la Norteamérica de Dennett, el poder de persuasión. Todo esto sin que la necesidad de lo "espiritual" en el hombre contemporáneo, así como su búsqueda e inquietud por lo trascendental, se reduzca. El agnosticismo parece ser una tendencia creciente de aquellos que, tomando distancia de las prácticas concretas de algún credo religioso, no profesan, sin embargo, una actitud combativa ateísta frente a la religión, sino que más bien mantienen una actitud expectante, esperando, en cierta forma, ser convencidos (o por lo menos "persuadidos") de una realidad que, pudiendo ser aprehendida, los jalone hacia el ámbito de lo trascendental. Como señala el teólogo español Andrés Torres Queiruga, la presencia de Dios en la conciencia humana es siempre oscura y ambigua, por eso la idea de Dios como una especie de arbitrio divino no tiene cabida (Torres, 2008, p. 255).

No obstante, solo la apelación a un principio trascendente podrá ofrecer el máximo fundamento que legitima y obliga incondicionalmente, afirma Gomez Caffarena (2007, p. 198). La presencia dinámica del hecho religioso, bien entendido, es capaz de otorgar las energías necesarias para colocarse más allá del éxito fugaz, del simple agrado, del egoísmo. Y de la pregunta por el cui bono. $\mathrm{Ni}$ la sola razón científica, ni tampoco el argumento de beneficio materialista o inmediato bastan para intentar comprender el fenómeno religioso en toda su extensión y en todas sus implicaciones. Como afirma Juan Antonio Estrada:

La mentalidad científica tiende al positivismo, a la búsqueda de hechos y comprobaciones, y se extiende a todos los ámbitos de la cultura. La postura científica de atenerse a lo empírico se transforma cuando se convierte en una tesis filosófica que niega la existencia divina. Que la metodología científica prescinda necesariamente de Dios, porque tiene que explicar los fenómenos por sí mismos, no equivale a la tesis de que no existe $(2015$, p. 70$)$.

Si bien el ser humano puede poseer la gran mayoría de las cualidades que lo hacen persona sin necesidad de recurrir a la creencia en Dios, también es cierto que en la medida en que ingresa en el tejido que va configurando el progreso cultural en el que se halla seguramente inserta alguna experiencia de religión, puede reconocer con claridad que la mejor manera de entender, proteger y mantener los valores auténticos que asume como fundamentales en su vida consiste en la aceptación de un fondo divino sustentador (Gómez, 2007, p. 201).

Finalmente, atendiendo a las consideraciones de Andrés Torres Queiruga, es posible apreciar que la persona humana, desde la emergencia determinada por el proceso evolutivo, adquiere su realidad, claridad, concreción y densidad. La persona sale del mundo, de la materialidad y de la animalidad, y emerge por encima del mundo de la cultura y de la libertad. Aquí se despliega su dinamismo, que, de suyo y en su realidad última, tiende a la comunión con Dios, y como tal puede ser diferenciado, recibiendo esa comunión su último sentido (Torres, 2008, p. 252). Como dice Manuel Fraijó, "la humanidad se merece que exista Dios; pero también se merece que no alumbremos precipitadamente lo divino" (1986, p. 257). 


\section{Referencias}

Ayala, F. J. (1991). Origen y evolución del hombre. España: Alianza.

Barbour, I. (1966). Issues in science and religion. Englewood Cliffs, NJ: Prentice-Hall

Bascompte, S. J. y Luque, S. B. (2012). Evolución y complejidad. España: Universidad de Valencia.

Beaman, L. y Tomlins, S. (2014). Atheist Identities. Spaces and Social Contexts. Londres: Springer.

Coulson, C. (1953). Christianity in a Age of Science. Londres: Oxford University Press.

Coulson, C. (1955). Science and Christian Belief. Londres: Oxford University Press.

De Lándazuri., O. C. (2008). Lo real y lo virtual en la neurociencia: ¿Inteligencia artificial o tránsito hacia una nueva metaciencia? Themata, 40, 127-132.

Dennett, D. C. (1990). The interpretation of texts, people and other artifacts. Philosophy and Phenomenological Research, 50, pp. 177-194.

Dennett, D. C. (1995/2000). Darwin's dangerous idea: evolution and the meaning of life. USA: Simon and Schuster (Traducción de C. Blanco-Morales. La peligrosa idea de Darwin. Evolución y significados de la Vida. Barcelona: Galaxia Gutenberg, 2000).

Dennett, D. C. (2003/2004). Freedom evolves. New York: Viking Penguin (Trad. de R. Vila Vernis. La evolución de la Libertad. Barcelona: Paidós, 2004.)

Dennett, D. C. (2005/2006). Dulces sueños: Obstáculos filosóficos para una ciencia de la conciencia (Traducción de J. Barba y S. Jawerbaum). Buenos Aires/Madrid: Katz, 221 pp. [título original: (2005). Sweet Dreams: Philosophical Obstacles to a Science of Consciousness. MIT Press: Cambridge, MA. P. 213].

Dennett, D. C. (2006/2007). Rompiendo el hechizo. La religión como un fenómeno natural (Traducción de F. de Brigard). Argentina: Katz (versión original 2006, Breaking the spell: Religion as a natural phenomenon. USA: Penguin).

Dennett, D. C. (2009, diciembre). The evolution of misbelief. Behavioral and Brain Sciences (32) 6, pp. 493-510.

Dennett, D. C. (2013). Intuition Pumps and Other Tools for Thinking. New York: W. W. Norton. 
Durkheim, É. (1982). Las formas elementales de la vida religiosa. El sistema totémico en Australia. Madrid: Akal.

Estrada, J. A. (2012). El sentido y el sinsentido de la vida. Madrid: Trotta.

Estrada, J. A. (2015). ¿Qué decimos cuando hablamos de Dios? La fe en la cultura escéptica. Madrid: Trotta.

Fagundes, S. (s. f.). Fundamentalismo científico: uma forma de pseudo-ciência. [Versión en línea]. Recuperado de http://www.ibamendes.com/2010/07/ fundamentalismo-cientifico-uma-forma-de.html

Ferrara, R. (2012). Religión y filosofía. España: Trotta.

Fraijó, M. (1986). El sentido de la historia: introducción al pensamiento de W. Pannenberg. Madrid: Cristiandad.

Fraijó, M. (1994). Filosofía de la religión: Estudios y Textos. Madrid: Trotta.

Giberson, K. y Artigas, M. (2007). Oracles of science. Celebrity scientists versus god and religion. New York: Oxford University Press.

Gómez, C. J. (1996). Interrogante: Dios. Madrid: Sal Terrae.

Gómez, C. J. (2007). El enigma y el misterio, una filosofía de la religión. Madrid: Trotta.

González, W. J. (2009). Evolucionismo: Darwin y enfoques actuales. La Coruña: Netbiblo.

Hamburger, J. (2003). La filosofía de las ciencias, hoy. México: Siglo XXI.

Küng, H. (1996). Ser cristiano. Madrid: Trotta.

Maldonado, C. C. (Ed.) (2012). ¿Qué son las ciencias de la complejidad? Filosofía de la ciencia de la complejidad. En C. Maldonado, Derivas de complejidad: fundamentos científicos y filosóficos (pp. 7-102). Bogotá: Universidad del Rosario.

Mühlin, M. (2011). Einstein und die Religion: Das Wechselverhältnis zwischen religiös-weltanschaulichen Gehalten und naturwissenschaftlicher Theoriebildung Albert Einsteins in seiner Entwicklung. Göttingen: Vandenhoeck \& Ruprecht.

Nietzsche, F. (1966). Jenseits von Gut und Böse. Vorspiel einer Philosophie der Zukunft. Munchen: Hanser.

Peacocke, A. (2008). Los caminos de la ciencia hacia Dios. El final de toda nuestra exploración. Madrid: Sal Terrae. 
Rahner, K. (1967). Schriften zur Theologie. Einsiedeln: Benziger.

Raven, C. (1953). Natural religion and Christian theology. Cambridge: Cambridge University Press.

San Martín, S. J. (2013). Antropología Filosófica I. De la Antropología científica a la filosófica. Madrid: UNED.

Schaeffler, R. (1983). Religionsphilosophie. Munich: Karl Alber.

Spencer, H. (1864). The principles of Biology. London: Williams and Norgate.

Taleb, N. N. (2008). El cisne negro. Barcelona: Paidós.

Thompson, D. L. (2009). Daniel Dennett. London: Continuum.

Torres, Q. A. (2008). Repensar la Revelación. La revelación divina en la realización humana. Madrid: Trotta.

Victoria, W. P. y Maestre, S. A. (2011). Los instrumentos del nuevo orden mundial: el derecho, la economía, la ciencia, el lenguaje y la religión en la sociedad del siglo XXI. Madrid: Universidad Complutense.

Wilson, B. R. (comp. \& ed.) (1970). Rationality. Londres: Harper \& Row.

Weinberg, S. (1996). The Quantum Theory of Fields. Cambridge: Cambridge University Press. 